\title{
Historical overview of planetary nebulae research
}

\author{
Sun Kwok \\ Faculty of Science, The University of Hong Kong, Hong Kong, China \\ email: sunkwok@hku.hk
}

\begin{abstract}
Planetary nebulae (PNs) were first discovered over 200 years ago and our understanding of these objects has undergone significant evolution over the years. Developments in astronomical optical spectroscopy and atomic physics have shown that PNe are gaseous objects photoionized by UV radiation from a hot central star. Studies of the kinematics of the nebulae coupled with progress in theories of stellar evolution have led to the identification that $\mathrm{PNe}$ are evolved stars and progenitors of white dwarfs. Development of infrared and millimeterwave technology in the 1970s made us realize that there is significant amount of neutral matter (molecules and dust) in PNe. The link of PNe to the stellar winds from their progenitor asymptotic giant branch (AGB) stars and subsequent dynamical interactions are now believed to be the underlying causes of the morphological structures of PNe. The role of PNe as prolific molecular factories producing complex molecules and organic solids has significant implications on the chemical enrichment of the Galaxy.

In this paper, we discuss the misconceptions and errors that we have encountered in our journey of understanding the nature of PN. The various detours and dead ends that had happened during our quest to pin down the evolutionary status and causes of nebulae ejection will be discussed. As there are still many unsolved problems in PN research, these lessons of history have much to offer for future progress in this field.
\end{abstract}

Keywords. Binaries: general, planetary nebulae: general, stars: AGB and post AGB, stars: evolution

\section{Introduction}

The study of planetary nebulae (PNe) began with the discovery of the first PN (M27, the Dumbbell Nebula) in 1764 by Charles Messier. The first step towards an understanding of the physical nature of PNe is the spectroscopic observation of NGC 6543 by William Huggins in 1864, showing that it is a gaseous nebula and is not a collection of stars. In 1922, from an observed correlation between the magnitude of the central star with the size of the nebula, Hubble suggested the the energy of the nebula is derived from the central star (Hubble 1922). With the advances in atomic physics in the early parts of the 20th century, Menzel (1926) applied atomic theories to come to the conclusion that the nebula is ionized by Lyman continuum photons emitted by a hot central star. Zanstra (1927) was able to quantitatively derive the temperature of the central star from the nebular $\mathrm{H} \beta$ flux and found out that the central stars of PNe are much hotter than normal stars. The most significant mystery of the unidentified nebular lines (proposed to be due to a new element nebulium) are in fact transitions from metastable states of $\mathrm{N}^{+}, \mathrm{O}^{+}$, and $\mathrm{O}^{++}$(Bowen 1928). These discoveries are the result of application of new physics and represents the beginning of the discipline of astrophysics.

Planetary nebulae research up to the 1970s can be grouped into three main areas: (i) the discovery of nebulous emission-line objects from imaging (Abell 1966, Perek \& Kohoutek 1967) and objective prism surveys (Minkowski 1964, Henize 1967); (ii) The employment 
S. Kwok

of spectroscopic techniques to study nebular physics (photoionization, recombination, collisional excitation) and the determination of elemental abundances (Aller 1956); (iii) the placement of PNe in the context of stellar evolution; and (iv) theoretical ideas on the formation of the nebula.

The purpose of a historical overview is not to give a comprehensive review of the subject but to use some examples of historical development to remind ourselves the tortuous paths that we went through in order to achieve progress. The goal is to show how advances were made in the past and hope that we can learn from the mistakes of the past and not to repeat them again.

\section{PN as a phase of stellar evolution}

In the early 20th century, stars were believed to evolve from high to low temperatures along the main sequence. PNe, having hot central stars, were believed to be young stars. However, Curtis (1918) found from the galactic distribution of PNe to belong to the old star population, therefore casting doubts on their young star status. The correct qualitative evolutionary status of PNe was first proposed by Shklovsky (1956a), who suggested that $\mathrm{PNe}$ were descendants of red giants and progenitors of white dwarfs. The fact that the observed expansion velocities of PNe were similar to the expected escape velocities of red giants led to the suggestion that the nebulae of PNe were ejected envelopes of red giants (Abell \& Goldreich 1966).

The next challenge was to place the central stars of PNe on the Hertzsprung-Russell (H-R) diagram, which has become the commonly accepted plot to trace the evolution of stars. The temperatures of central stars can be derived from the Zanstra method, and the apparent brightness of PN can be estimated from the apparent magnitudes of the central stars. Using the Shklovsky method of distance determination (Shklovsky 1956b), the luminosities of the central stars could be obtained. This has led to the evolutionary tracks of PNe known as the Harman-Seaton sequence (O'Dell 1963, Harman \& Seaton 1966). The Harman-Seaton sequence suggests that PNe are formed at the end of the horizontal branch, followed by a rapid rise in temperature and luminosity (Roxburgh 1967). After the publication of Roxbourgh's paper, the editorial of Nature commented "Theoretical models agree tolerably well with the observations... the overall properties of planetary nebulae are now well understood" (Nature, September 30, 1967).

This celebration of success was premature. The shape of the Harman-Seaton sequence caused great difficulties for the theoreticians as the evolutionary time is constrained by the dynamical time of the nebula $\left(\sim 10^{4} \mathrm{yr}\right)$ and it is difficult for the luminosity and temperatures of a star to change so drastically over such a short time (Salpeter 1968, Deinzer \& Hansen 1968; Kutter, Savedoff, \& Schuerman 1969; Shaviv 1978).

It was only many years later that we realized that it was the observations which were at fault. The high temperatures of the central stars means that they are often faint in the visible and difficult to measure. The optical brightness of the central star is often contaminated by nebular emission. There is a significant problem of missing fluxes as the result of leaking UV photons (nebulae not ionization bounded), and the neglect of significant amount of fluxes emitted in the infrared. Furthermore, the Shklovsky method of distance determination is not reliable (Kwok 1985). A review of the historical development was given by Schönberner (1993).

It is a rare case in astronomy history that the situation was saved by a simple theory. Paczyński $(1970,1971)$ started with a static model of AGB stars with different amount of masses in the hydrogen envelope. He noticed that the star will not evolve to the blue until the envelope mass $\left(M_{\mathrm{H}}\right)$ drops to a very low value $\left(M_{\mathrm{H}}<10^{-3} \mathrm{M}_{\odot}\right.$ for a core mass 
of $\left.0.6 \mathrm{M}_{\odot}\right)$. After the envelope mass drops below this value, the star will begin to move to the left side of the H-R diagram horizontally, as the core mass is hardly changed by H-burning of the very thin shell. Instead of the Harman-Seaton sequence, central stars of PNe evolve at constant luminosity across the H-R diagram.

The Paczyński model has become the modern model of PN evolution. PN central stars generate energy by $\mathrm{H}$-shell burning above an electron-degenerate $\mathrm{C}-\mathrm{O}$ core. Increases in the effective temperatures of the central star is the result of the thinning of the $\mathrm{H}$ envelope (and mass loss). All stars with different core masses evolve horizontally until the $\mathrm{H}$ fuel is depleted, and the time to cross the H-R diagram is highly dependent on the core mass. The Paczyński model was extended by many subsequent works, including those of Schönberner (1979, 1981), Kovetz \& Harpaz (1981), Iben (1984), Wood \& Faulkner (1986), and Vassiliadis \& Wood (1993).

The Paczyński model also raises new questions on the existence of PNe. Since the existence of PNe requires the dynamical age of the nebula be comparable to the evolutionary age of the central star, and the central star evolutionary time is highly dependent on the core mass, this implies that high-mass PNe will have too short of life time to be observed, and low-mass PN will have their nebulae dissipated before the onset of photoionization. We have come to realize that the PN phenomenon is a time-dependent dynamical system.

\section{Formation of the nebulae}

The next problem to tackle was the ejection of the nebulae. Intuitively, this seems simple enough. The surface gravity of a red giant star is low and it should not be too difficult to let go of its $\mathrm{H}$ envelope. Many models have been proposed over the years. These include ejection as the result of dynamical instabilities (Roxburgh 1967, Lucy 1967, Paczyński and Ziolkowski 1968); pulsational instabilities (Kutter \& Sparks, 1974, Wood 1974, Tuchman et al. 1979); envelope oscillations due to thermal instabilities in the core (Smith and Rose 1972); radiation pressure (Faulkner 1970, Finzi and Wolf 1971, Finzi et al. 1974), and thermal pulses (Härm \& Schwarzschild 1975, Trimble \& Sackman 1978). All these sudden-ejection models suffer from a common problem that they either eject too much or too little mass. Paczyński showed that the central star will not move in the H-R diagram until the envelope mass fall below $10^{-3} \mathrm{M}_{\odot}$, and no sudden ejection mechanism can be so precise in leaving behind this exact amount of mass above the core. If the envelope is indeed ejected, then the ejected mass will disperse into the interstellar medium without ever being illuminated as the central star has not evolved far enough to photoionize it.

There is also the problem of backfill. It was realized that additional pressure is needed to maintain the expansion of the nebula and prevent it from falling back onto the central star (Capriotti 1973; Wentzel 1976; Ferch \& Salpeter 1975). In order to avoid backfill, the ejected envelope has to be supported by a stellar wind (Mathews 1966) or pressure from photoionization heating (Sofia \& Hunter 1967).

The puzzle of nebular ejection was only solved after the discovery of AGB mass loss, which manifests itself through molecular emission in the millimeter wavelengths and dust emissions in the infrared. Thousands of AGB stars have now been observed to be undergoing large-scale, steady mass loss over the last few hundred thousand years of their lives. These mass loss remove the $\mathrm{H}$ envelope and gradually expose the hot core. The amount of mass ejected can be up to several solar masses and is much larger than the observed mass in the ionized nebula. These massive circumstellar envelopes must have an effect on the formation of PN. What we needed is a mechanism to compress these circumstellar materials into a shell and accelerate it to higher expansion speeds. This was 
provided by the mechanism of interacting stellar winds (ISW, Kwok et al. 1978; Kwok 1982).

The ISW model makes three predictions: (i) that fast winds from central stars are common in PNe; (ii) the remnant of the AGB envelopes can be observed in PNe; (iii) the shocked stellar wind bubble can be observed in X-rays. The first prediction was soon confirmed with the launch of the IUE satellite, where fast winds of velocities 2000-4000 $\mathrm{km} \mathrm{s}^{-1}$ are detected through the P-Cygni profiles of resonance lines in the UV (Heap et al. 1978; Perinotto 1993). After the introduction of CCD as optical imaging devices, optical haloes are found to be common in PNe (Jewitt et al. 1986; Chu et al. 1987; Corradi et al. 2003). The launch of the IRAS satellite has resulted in the detection of over $1000 \mathrm{PNe}$ in the far infrared, showing the presence of a strong dust component in PNe (Pottasch et al. 1984, Zhang \& Kwok 1991). This dust component, as well as molecular emissions, provide direct evidence for the link between PNe and the circumstellar envelopes of AGB stars. Extended, soft X-ray continuum emission was detected in PNe by the ROSAT, Chandra, and XMM X-ray satellites (see Guerrero, these proceedings).

$\mathrm{PNe}$ are dynamical systems whose evolution is tightly coupled with the evolution of the central stars. Consistent treatment of the dynamics and evolution including the effects of time-dependent photoionization have led to the natural explanation of the morphological structures of PNe such as crowns, haloes, and multiple shells (Schmidt-Voigt \& Köppen 1987a,b; Marten \& Schönberner 1991; Steffen et al. 1998; Corradi et al. 2000; Perinotto et al. 2004).

\section{Binary evolution}

One year before Paczyński made his presentation on PN evolution at the IAU Symposium on planetary nebulae in Ithaca in 1976, he presented a paper on "common envelope binaries" in IAU Symposium 73 on "Structure and Evolution of Close Binary Systems" in Cambridge (Paczyński 1976). Paczyński outlined a number of scenarios of binary evolution if one component is able to transfer mass to its companion and influence its evolution. This sparked the gold age of binary star research, which was initiated by the discovery that novae are binary systems (Kraft 1964). The possibility that a binary system can undergo mass transfer through either Roche Lobe or stellar wind accretion while either the primary or the secondary is on the post-main-sequence phase of evolution has opened up the phenomenon of cataclysmic variables (CVs, Warner 2003). Over 100 types of binaries and products of their evolution have been calculated and it is estimated that binary evolution can generate evolutionary trees of hundreds of branches (Tutukov \& Yungelson 2002). It was also realized that close binary systems can form common envelopes, resulting in common envelope ejections (Paczyński 1976; Webbink 1979; van den Heuvel 1976, Iben \& Tutukov 1985, 1987). The possible relevance of common envelope ejection with PNe was investigated by many authors, with many possible variations and scenarios possible (Iben \& Tutukov 1989, de Kool 1990).

Recently, it has been proposed that $\mathrm{PN}$ is a $\mathrm{CV}$ phenomenon based on the fact that some PNe have binary central stars (de Marco 2010). If indeed some objects in the PN catalog are CVs, then we have to address the question what kind of CVs are they? How did they evolve to this stage and what they will evolve into in the future? More specifically, where do these objects fit into the tree of binary star evolution?

It is indeed quite likely that there are objects in the PN catalog which are missclassified. The most common confusion is with symbiotic stars (Allen 1984, 1988). Symbiotic stars share many of the observational properties of PNe. They have an emission line spectrum, infrared excess, and some times X-ray continuum emission. The evolution 
of a symbiotic star is a competition between mass transfer and H-shell burning. If the rates are comparable, the binary is a symbiotic star. If the accretion rate is greater, they are nova candidates (Paczyński \& Rudak 1980). Clearly, symbiotic stars is a well-defined binary phenomenon (Yungelson et al. 1995) and it should be possible to sort out whether a specific object in the PN catalog is a symbiotic star or not.

It is certainly possible that a star that has evolved to become a white dwarf (through the PN phase) can later be re-ignited by mass transfer to become a nova or a symbiotic star. So we can say that both novae and symbiotic stars are post-PN phenomena.

Since the observational properties of $\mathrm{PNe}$ are not unique, we cannot define $\mathrm{PNe}$ by their observational properties. As in any other astrophysical phenomenon, one progresses from an observational definition to a theoretical one as our understanding of the physical processes matures. Since there are red giants and there are white dwarfs, there must be a way for a red giant to evolve to become a white dwarf. I would therefore propose to define a $\mathrm{PN}$ as an ionized circumstellar shell showing some degree of symmetry surrounding a hot, compact star evolving from the AGB to white dwarf phases. If there are objects in the PN catalog that are there as the result of other paths of evolution, they should be labeled as such and not as a PN.

\section{Morphology of planetary nebulae}

The morphological classification of PNe began with Curtis (1918) who put PNe into helical, annular, disk, and amorphous groups. The catalogue of Perek \& Kohoutek (1967) classified PNe into stellar, disk, irregular, ring, anomalous. In the CCD era, PNe are classified into elliptical, bipolar, point-symmetric, irregular, and stellar (Stanghellini et al. 1993). These are geometric descriptions of the apparent 2-D structures and do not necessarily reflect the true $3-\mathrm{D}$ structures of PNe. These schemes are subject to sensitivity dependence, species dependency, and projection effects.

Since the circumstellar envelopes of AGB stars are generally spherical, the transformation into asymmetric shapes of PN must have occurred within the few thousand years of post-AGB evolution. How PNe are shaped into asymmetric structures is one of the most fascinating areas of PN research (Meixner et al. 2004, Corradi et al. 2007, Zijlstra et al. 2010).

Although our perception of the morphology of $\mathrm{PNe}$ is dominated by their optical images, we should remember that the ionized component (from which the optical emission line arises) is not where most of the mass of $\mathrm{PNe}$ reside. Most of the masses of $\mathrm{PNe}$, in particular the younger ones, are in the neutral (molecular and dust) components. Many bipolar nebulae (e.g., NGC 2346 and NGC 6302) are clearly confined by external media which do not emit visible light. The bright lobes of PN may represent actual cavities in the density structure where the density is low, and the gas can be photoionized. If this is the case, then the bright lobes are not the result of mass ejection events but the result of illumination (Kwok 2010).

The old morphological classification problem is therefore not a simple one. As the nebula expands and the central star evolves to higher temperatures, the morphology will evolve accordingly. A bipolar nebula ionization bounded in the equatorial region by a massive neutral envelope will become spherical in shape as the nebula is fully ionized. A re-construction of the full 3-D mass structure of $\mathrm{PNe}$ will require multi-wavelength observations and sophisticated simulation tools. 


\section{Conclusions}

I hope the above historical discussions illustrate that the paths to an understanding of PN have not been easy. Whatever we have achieved today is the result of collective hard work and wisdom of many people.

Planetary nebulae research has rejuvenated itself several times in the last 200 years. From its origin in the catalogs of nebulous objects, it became the laboratory of astrophysics in the 1920-1960s. Although a qualitative evolutionary scenario was proposed in 1956, the quantitative physical description of PN evolution was only developed in the 1970s. The introduction of infrared and millimeter-wave techniques led to the discovery of mass loss in AGB stars, leading to the realization of the role AGB mass loss plays in the formation of the nebulae. Studies of nebular evolution became a laboratory of dynamical theories and the lessons learned in PN research were applied to Wolf-Rayet nebulae, young stellar objects, and active galactic nuclei. The high-dynamic range optical imaging by CCDs, in particular with the HST, have allowed detailed morphological studies of PNe, leading to the realization of their complex structures.

Modern optical, infrared, UV, and X-ray spectroscopic studies of PNe have led to the detection and identification of thousands of atomic lines and hundreds of molecular lines. However, there still remain a number of unidentified (probably solid-state) emission features. We now appreciate that $\mathrm{PNe}$ are galactic factories of complex organics and $\mathrm{PNe}$ are likely to play a role in the chemical enrichment of the early solar system (Kwok 2004).

PNe play a major role in our understanding of nucleosynthesis and the abundance of chemical elements. They are also used as a tool to study the chemical evolution and star formation history of galaxies. PNe have also been used as standard candles to determine the extragalactic distance scales and the distribution of dark matter in galaxies.

Whether PN research has a future depends on whether this community can find new angles to look at the old problems, and whether we can create new problems from new observational techniques. The number of observational instruments available to us today are much more versatile and powerful than in the past. For the first time we have the ability to perform spectroscopic and imaging observations of PNe throughout the electromagnetic spectrum almost continuously from radio to X-ray. It is up to us to properly synthesize these observations and derive an integrated picture of the structure of PNe in order to achieve an understanding of their origin and evolution.

\section{Acknowledgements}

I would like to dedicate this review to my good friend Mario Perinotto, who has devoted his life for the pursuit of planetary nebulae research. This work was supported by a grant from the Research Grants Council of the Hong Kong Special Administrative Region, China (Project No. HKU 7031/10P).

\section{References}

Abell, G. O. 1966, ApJ, 144, 259

Abell, G. O. \& Goldreich, P. 1966, PASP, 78, 232

Allen, D. A. 1984, Proc. Astr. Soc. Australia, 5, 369

Allen, D. A. 1988, in IAU Colloq. 103, The Symbiotic Phenomenon, eds. J. Mikolajewska et al. (Reidel:Dordrecht), p. 3

Aller, L. H. 1956, Gaseous Nebulae, (New York: Wiley)

Bowen, I. S. 1928, ApJ, 67, 1

Capriotti, E. R. 1973, ApJ, 179, 495

Chu, Y., Jacoby, G. H., \& Arendt, R. 1987, ApJS, 64, 529 
Corradi, R. L. M., Schönberner, D., Steffen, M., \& Perinotto, M. 2000, A\&SA, 354, 1071

Corradi, R. L. M., Schönberner, D., Steffen, M., \& Perinotto, M. 2003, MNRAS, 340, 417

Corradi, R. L. M., Manchado, A., \& Soker, N. (eds.) 2007, Asymmetric Planetary Nebulae IV, eds. IAC electronic publications

Curtis, H. D. 1918, Publ. Lick Obs., Vol. XIII, Part III, p. 57

Deinzer, W. \& Hansen, C. 1968, AJ, 73, 173

de Marco, O. 2010, PASP, 121, 316

de Kool, M. 1990, ApJ, 358, 189

Faulkner, D. J. 1970, ApJ, 162, 513

Ferch, R. L. \& Salpeter, E. E. 1975, ApJ, 202, 195

Finzi, A. \& Wolf, R. A. 1971, $A \mathscr{G} A, \mathbf{1 1}, 418$

Finzi, A., Finzi, R., \& Shaviv, G. 1974, A\&A, 37, 325

Härm, R. \& Schwarzschild, M. 1975, ApJ, 200, 324

Harman, R. J. \& Seaton, M. J. 1966, MNRAS, 132, 15

Heap, S. R. et al., 1978, Nature, 275, 385

Henize, K. G. 1967, ApJS, 14, 125

Hubble, E. 1922, ApJ, 56, 400

Iben, I. 1984, ApJ, 277, 333

Iben, I., Jr. \& Tutukov, A. V. 1985, ApJS, 58, 661

Iben, I., Jr. \& Tutukov, A. V. 1987, ApJ, 313, 727

Iben, I., Jr. \& Tutukov, A. V. 1989, in IAU Symp. 131: Planetary Nebulae, ed. Torres-Peimbert, (Kluwer), p. 505

Jewitt, D. C., Danielson, G. E., \& Kupferman, P. N. 1986, ApJ, 302, 727

Kovetz, A. \& Harpaz, A. 1981, A\& A, 95, 66

Kraft, R. P. 1964, ApJ, 139, 457

Kutter, G. S. \& Sparks, W. M. 1974, ApJ, 192, 447

Kutter, G. S., Savedoff, M. P., \& Schuerman, D. W. 1969, ApSS, 3, 182

Kwok, S. 1982, ApJ, 258, 280

Kwok, S. 1985, ApJ, 290, 568

Kwok, S. 2004, Nature, 430, 985

Kwok, S. 2010, PASA, 27, 174

Kwok, S., Purton, C. R., \& FitzGerald, M. P. 1978, ApJ, 219, L125

Lucy, L. B. 1967, AJ, 72, 813

Marten, H. \& Schönberner, D. 1991, A\&SA, 248, 590

Mathews, W. G. 1966, ApJ, 143, 173

Meixner, M., Kastner, J., Balick, B., \& Soker, N. (eds.) 2004, Asymmetric Planetary Nebulae III, ASP conf. ser., Vol. 313

Menzel, D. H. 1926, PASP, 38, 295

Minkowski, R. 1964, PASP, 76, 197

O'Dell, C. R. 1963, ApJ, 138, 67

Paczyński, B. 1970, Acta Astr. 20, 47

Paczyński, B. 1971, Acta Astr., 21, 417

Paczyński, B. 1976, in IAU Symp. 73: Structure and Evolution of Close Binary Systems, P. Eggleton, S. Mitton, S., \& J. Whelan (eds), (Reidel:Dordrecht), p. 75

Paczyński, B. \& Rudak, B. 1980, A\& A, 82, 349

Paczyński, B. \& Ziólkowski, J. 1968, Acta Astr., 18, 255

Perek, L. \& Kohoutek, L. 1967, Catalog of Galactic Planetary Nebulae, (Academia: Praha)

Perinotto, M. 1993, in IAU Symp 155: Planetary Nebulae, eds. R. Weinberger \& A. Acker (Kluwer:Dordrecht), p. 57

Perinotto, M., Schönberner, D., Steffen, M., \& Calonaci, C. 2004, A\&A, 414, 993

Pottasch, S. R., et al. 1984, AधA, 138, 10

Roxburgh, I. W. 1967, Nature, 215, 838

Salpeter, E. E. 1968, IAU Symp. 34: Planetary Nebulae, eds. D. Osterbrock \& C.R. O'Dell (Reidel:Dordrecht), p. 409 
Schmidt-Voigt, M. \& Köppen, J. 1987a, A\&A, 174, 211

Schmidt-Voigt, M. \& Köppen, J. 1987b, A\& A, 174, 223

Schönberner, D. 1979, A\&\&A, 79, 108

Schönberner, D. 1981, A\&广A, 103, 119

Schönberner, D. 1993, Acta Astron., 43, 297

Shaviv, G. 1978, IAU Symp 76: Planetary Nebulae, ed. Y. Terzian, (Reidel:Dordrecht), p. 195

Shklovsky, I. 1956a, Astr. Zh., 33, 315

Shklovsky, I. 1956b, Astr. Zh., 33, 222

Smith, R. L. \& Rose, W. K. 1972, ApJ, 176, 395

Sofia, S. \& Hunter, J. H. 1967, AJ, 72, 830

Stanghellini, L., Corradi, R. L. M., \& Schwarz, H. E. 1993, A\&AS, 279, 521

Steffen, M., Szczerba, R., \& Schönberner, D. 1998, A\&\&A, 337, 149

Trimble, V. \& Sackman, I. J. 1978, MNRAS, 182, 97

Tuchman, Y., Sack, N., \& Barket, Z. 1979, ApJ, 234, 217

Tutukov, A. V. \& Yungelson, L. R. 2002, Astronomy Reports, 46, 667

van den Heuvel, E. P. J. 1976, in IAU Symp. 73:Structure and Evolution of Close Binary Systems, ed. P. Eggleton, S. Mitton, S., \& J. Whelan, (Reidel:Dordrecht), p. 35

Vassiliadis, E. \& Wood, P. R. 1993, ApJ, 413, 641

Warner, B. 2003, Cataclysmic Variable Stars, Cambridge University Press, Cambridge, pp. 592

Webbink, R. F. 1979, in IAU Colloq. 53: White Dwarfs and Variable Degenerate Stars, eds. H. van Horn, \& V. Weidemann, p. 426

Wentzel, D.G. 1976 ApJ, 204, 452

Wood, P. R. 1974, ApJ, 190, 609

Wood, P. R. \& Faulkner, D. J. 1986, ApJ, 307, 659

Yungelson, L., Livio, M., Tutukov, A., \& Kenyon, S. J. 1995, ApJ, 447, 656

Zanstra, H. 1927, ApJ, 65, 50

Zhang, C. Y. \& Kwok, S. 1991, A\&A A, 250, 179

Zijlstra, A. A., Lykou, F., McDonald, I., \& Lagadec, E. (eds.) 2010, Asymmetric Planetary Nebulae $V$, Jodrell Bank Centre for Astrophysics 